\title{
Subharmonic gap structure in short ballistic graphene junctions
}

\author{
J.C. Cuevas ${ }^{1,2,3}$ and A. Levy Yeyati ${ }^{1}$ \\ ${ }^{1}$ Departamento de Física Teórica de la Materia Condensada, \\ Universidad Autónoma de Madrid, E-28049 Madrid, Spain \\ ${ }^{2}$ Institut für Theoretische Festkörperphysik, Universität Karlsruhe, D-76128 Karlsruhe, Germany \\ ${ }^{3}$ Forschungszentrum Karlsruhe, Institut für Nanotechnologie, D-76021 Karlsruhe, Germany
}

(Dated: February 6, 2008)

\begin{abstract}
We present a theoretical analysis of the current-voltage characteristics of a ballistic superconductor-normal-superconductor (SNS) junction, in which a strip of graphene is coupled to two superconducting electrodes. We focus in the short-junction regime, where the length of the strip is much smaller than superconducting coherence length. We show that the differential conductance exhibits a very rich subharmonic gap structure which can be modulated by means of a gate voltage. On approaching the Dirac point the conductance normalized by the normal-state conductance is identical to that of a short diffusive SNS junction.
\end{abstract}

PACS numbers: 74.45.+c,74.50.+r,73.23.Ad,74.78.Na

Introduction.- Recently it has become possible to manipulate graphene, a single atomic layer of carbon, and to establish electrical contacts with it 1.2.3. This has opened the possibility of studying its transport properties, which are determined by the dynamics of two-dimensional Dirac fermions (massless excitations governed by a relativistic wave equation) $)^{2.3}$. Moreover, the fabrication of a new class of hybrid structures in which superconductors are coupled via graphene is now feasible ${ }^{\underline{4}}$. It has already been shown theoretically that the nature of the low-lying graphene excitations might lead to unexpected features in transport properties of these heterostructures ${ }^{5.6}$. Thus for instance, Titov and Beenakker ${ }^{6}$ have recently demonstrated that a SNS junction with an undoped strip of graphene as a normal region can sustain a supercurrent and it exhibits an unusual "quasi-diffusive" scaling of the dc Josephson effect.

The supercurrent in graphene hybrid systems may nicely reveal the interplay of superconductivity and the relativistic quantum dynamics of the electrons in these carbon structures. However, a direct comparison of the supercurrent between theory and experiment is usually not straightforward. This is due to the fact that the superconducting phase is prone to both quantum and thermal fluctuations, which depend on the electromagnetic environment in which the junction is embedded ${ }^{7}$. Thus, unless the environment is carefully designed, which is not an easy task, the supercurrent might be greatly reduced as compared with the theoretical predictions for idealized situations. For this reason, we propose to look at the current-voltage characteristics (I-V) of graphene SNS contacts, which do not suffer from the problems just mentioned. The main feature of the I-V curves of SNS junctions is the appearance of the so-called subharmonic gap structure, which consists of a series of conductance maxima at voltages $2 \Delta / n e$, where $n$ is an integer and $\Delta$ is the energy gap of the electrodes. This structure originates from the ocurrence of multiple Andreev reflections (MARs) $\underline{\underline{8}}$. The microscopic theory of these tunneling processes developed in the 1990's 9.10 has been shown to describe accurately the I-V characteristics of superconducting atomic point-contacts11.

In this Communication we present a theoretical study of the I-V characteristics of SNS junctions, where the normal region is a ballistic strip of graphene. We consider the experimentally relevant case of a short junction, in which the length of the strip is smaller than the superconducting coherence length $\xi$. We show that the conductance exhibits a pronounced subharmonic gap structure that can be tuned with a gate voltage. In the case of zero gate voltage, i.e. at the Dirac point, the I-V curves are, after rescaling, identical to those of a short diffusive SNS junction, which is a new consequence of the relativistic dynamics of the electrons in graphene.

Model for the graphene junction.- We consider the system shown schematically in Fig. 1 (upper panel), where a graphene strip is coupled to two superconducting electrodes. We also assume that an additional gate electrode allows to control the carrier concentration in the normal part of the junction. We focus here in the case where the width of the graphene strip $W$ is much larger than the junction length $L$ (in this case the details of the microscopic description of the strip edges become irrelevant). Our main goal is the analysis of the superconducting $\mathrm{I}-\mathrm{V}$ characteristics in the experimentally most relevant short-junction regime where $L$ is small relative to $\xi$. In terms of energy scales, this condition requires $\Delta \ll \hbar v / L$, where $v$ is the energy-independent electron velocity in graphene. It has been shown that, as long as the normal transmission coefficients do not depend on energy on the scale of $\Delta$, the transport properties of a short SNS junction can be expressed as a sum of independent contributions from individual conduction modes 12 . Therefore, the transport properties depend only on the distribution of transmission probabilities $\tau_{i}$ of these modes in the normal state. For describing the transmission distribution of a graphene junction one can adopt the model introduced in Ref. 13, which is briefly discussed in the next paragraphs.

The authors of Ref. 13 considered the junction depicted 

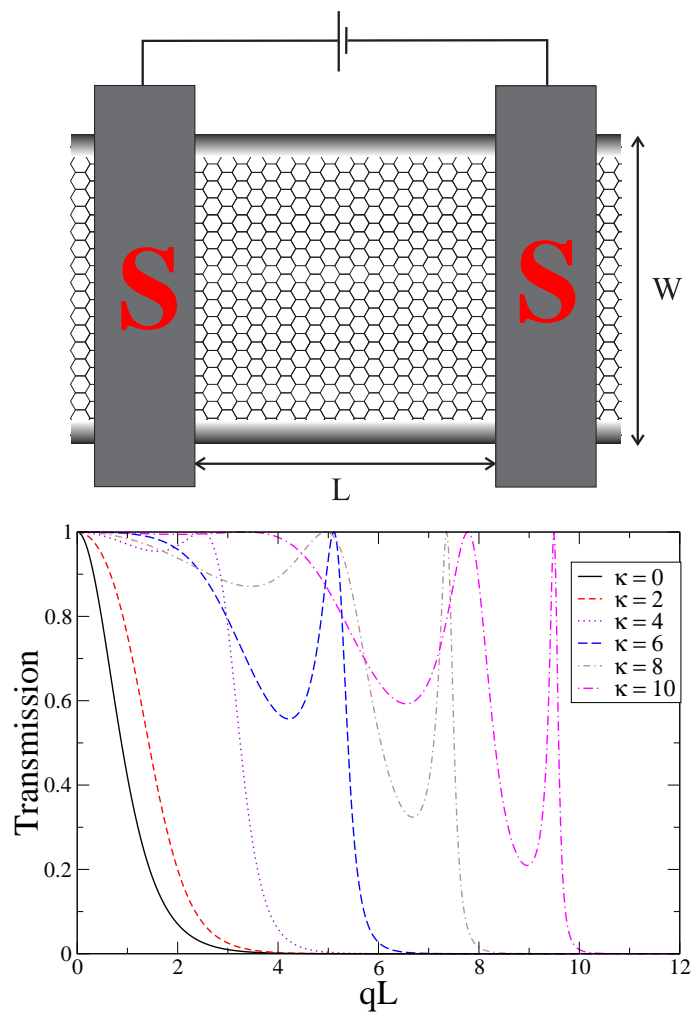

FIG. 1: (Color online) Upper panel: Schematic of a strip of graphene of width $W$, contacted by two superconducting electrodes (black rectangles) at a distance $L$. A voltage source drives a current through the strip. A separate gate electrode (not shown) allows the carrier concentration in the strip to be tuned around the neutrality point. Lower panel: Normal transmission of the conduction channels of the graphene junction as a function of the transversal momentum in the limit $W / L \gg 1$. The different curves correspond to different values of the dimensionless gate voltage $\kappa=e\left|V_{\text {gate }}\right| L / \hbar v$.

in Fig. 1 (upper panel). They determined the electron wave functions by solving the Dirac equation for massless fermions. In particular, they showed that assuming "infinite mass" boundary conditions at the strip edges $y=0$ and $y=W$, the transversal momenta are quantized as

$$
q_{n}=\frac{1}{W} \pi\left(n+\frac{1}{2}\right), \quad n=0,1,2, \ldots,
$$

with $n$ labeling the modes. Each mode has a twofold valley degeneracy.

In the model of Ref. 13, the gate voltage enters in the Dirac equation as an electrostatic potential. This potential $V(x)=V_{\text {gate }}$ for $0<x<L$ determines the concentration of the carriers in the strip. The value $V_{\text {gate }}=0$ corresponds to charge neutrality, being the point where electron and hole excitations are degenerate (known as the Dirac point). The electrodes are modelled by taking a large value $V(x)=V_{\infty}$ in the leads $x<0$ and $x>L$. (The parameter $V_{\infty}$ drops out of the results, if
$\left.\left|V_{\infty}\right| \gg\left|V_{\text {gate }}\right|.\right)$ The number of propagating modes $N$ is given by $N=\operatorname{Int}\left(k_{\infty} W / \pi+\frac{1}{2}\right)$, with $e\left|V_{\infty}\right|=\hbar v k_{\infty}$. We are interested in the limit $\left|V_{\infty}\right| \rightarrow \infty$ of an infinite number of propagating modes in the leads. By matching the solutions of the Dirac equation in the three regions of the junction one finds that the transmission probabilities at the Fermi level are given by ${ }^{13}$

$$
\tau_{n}=\left|\frac{2 \delta^{2}-2\left(q_{n}-k_{n}\right)^{2}}{e^{k_{n} L}\left(q_{n}-k_{n}+i \delta\right)^{2}+e^{-k_{n} L}\left(q_{n}-k_{n}-i \delta\right)^{2}}\right|^{2}
$$

with $\delta=e\left|V_{\text {gate }}\right| / \hbar v$ and $k_{n}=\sqrt{q_{n}^{2}-\delta^{2}}$ for $q_{n}>\delta$ or $k_{n}=i \sqrt{\delta^{2}-q_{n}^{2}}$ for $q_{n}<\delta$. These transmssion coefficients are plotted in Fig. 1] (lower panel) for different values of the dimensionless gate voltage $\kappa=e\left|V_{\text {gate }}\right| L / \hbar v$.

The normal-state conductance $G_{\mathrm{N}}$ of the junction and the corresponding resistance $R_{\mathrm{N}}$ are given by

$$
G_{\mathrm{N}}=R_{N}^{-1}=\frac{4 e^{2}}{h} \sum_{n=0}^{\infty} \tau_{n}
$$

Superconducting I- $V$ characteristics.- We now turn to the analysis of the current-voltage characteristics in the case in which the electrodes are in the superconducting state. As explained above, the current in the shortjunction limit $(L \ll \xi)$ can be expressed as a sum of independent channel contributions as follows

$$
I(V, t)=2 \sum_{n=0}^{\infty} I\left(V, t, q_{n}\right)
$$

where $t$ is the time and $I\left(V, t, q_{n}\right)$ is the single-channel current of a superconducting point-contact of transmission $\tau_{n}\left(q_{n}\right)$ (cf. Eq. 2). We compute this single-channel current using the Hamiltonian approach described in Ref. 10. Let us remind that in the case of a superconducting junction at finite voltage, the current oscillates on time as $I(V, t)=\sum_{m} I_{m}(V) \exp [i m \phi(t)]$, where $\phi(t)$ is the time-dependent superconducting phase difference given by the Josephson relation $\partial \phi(t) / \partial t=2 e V / \hbar$. We only consider here the dissipative dc current, which we shall simply denote as $I$ from now on. Moreover, in the limit $L \ll W$ considered here, one can replace the sum over $n$ in the previous equation by an integration.

We show in Fig. 2 the zero-temperature I-V characteristics for different values of the gate voltage. Notice that in the upper panel the current is expressed in an absolute scale, while in the lower one it is normalized by the normal-state resistance that scales as $R_{\mathrm{N}} \propto L$. At zero gate voltage, i.e. at the Dirac point, the current is formally identical to that of a short diffusive SNS junction ${ }^{12}$. This is easy to understand with the help of Eq. 2] From this expression one can show that for $V_{\text {gate }}=0$ the transmission distribution adopts the form $\rho(\tau)=(W / \pi L) 1 / 2 \tau \sqrt{1-\tau}$, which corresponds to the well-known bimodal distribution for diffusive wires 14 . Away from the Dirac point, the non-linearities of the I-V 


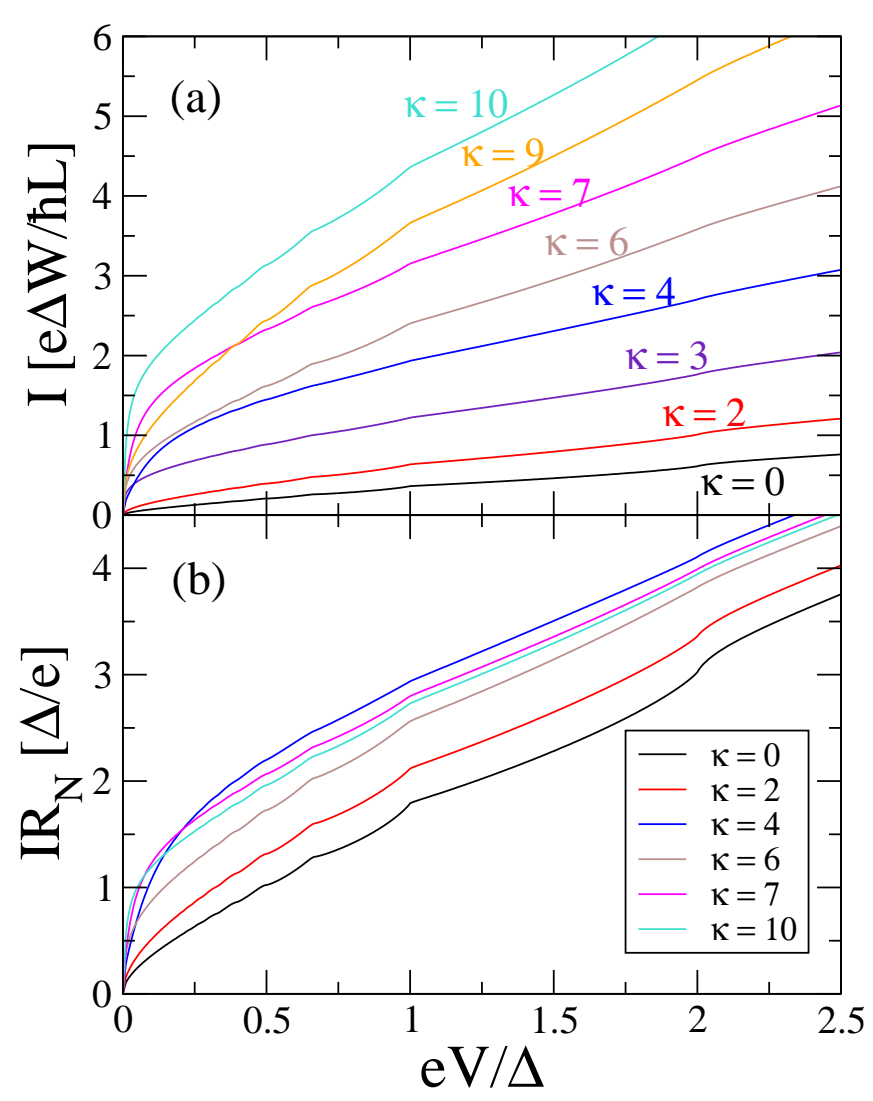

FIG. 2: (Color online) (a) Zero-temperature current-voltage characteristics of a superconducting ballistic graphene junction (length $\mathrm{L}$ short compared to the width $\mathrm{W}$ and the superconducting coherence length $\xi$ ), for different values of the gate voltage in the normal region $\left(\kappa=e\left|V_{\text {gate }}\right| L / \hbar v\right)$. (b) The same as in panel (a), but the current is normalized by the resistance of the junction in the normal state $R_{\mathrm{N}}$.

curves are modulated by the gate voltage. In particular, for gate voltages in which most of the open channels have a high transmission (see Fig. 10), the I-V curves are rather smooth (see for instance the curve for $\kappa=4$ ). Notice also that contrary to the normal state, the current at certain voltages below the gap is not neccesarily a monotonously increasing function of the gate voltage. This peculiarity is due to the fact that the subgap current is a very non-linear function of the transmission coefficients.

The non-linearities in the current are better observed in the differential conductance, which is shown in Fig. [3 The conductance exhibits a very pronounced subgap structure, although the maxima not always appear exactly at the voltages $2 \Delta / n e$ ( $n$ integer). As explained in the introduction, these peaks are due to the opening of new MAR processes at those voltages ${ }^{8}$. The pronounced peaks (especially at $2 \Delta / e$ and $\Delta / e$ ) come from the contribution of the low-transmitting conduction channels. This explains why this structure is more pronounced close

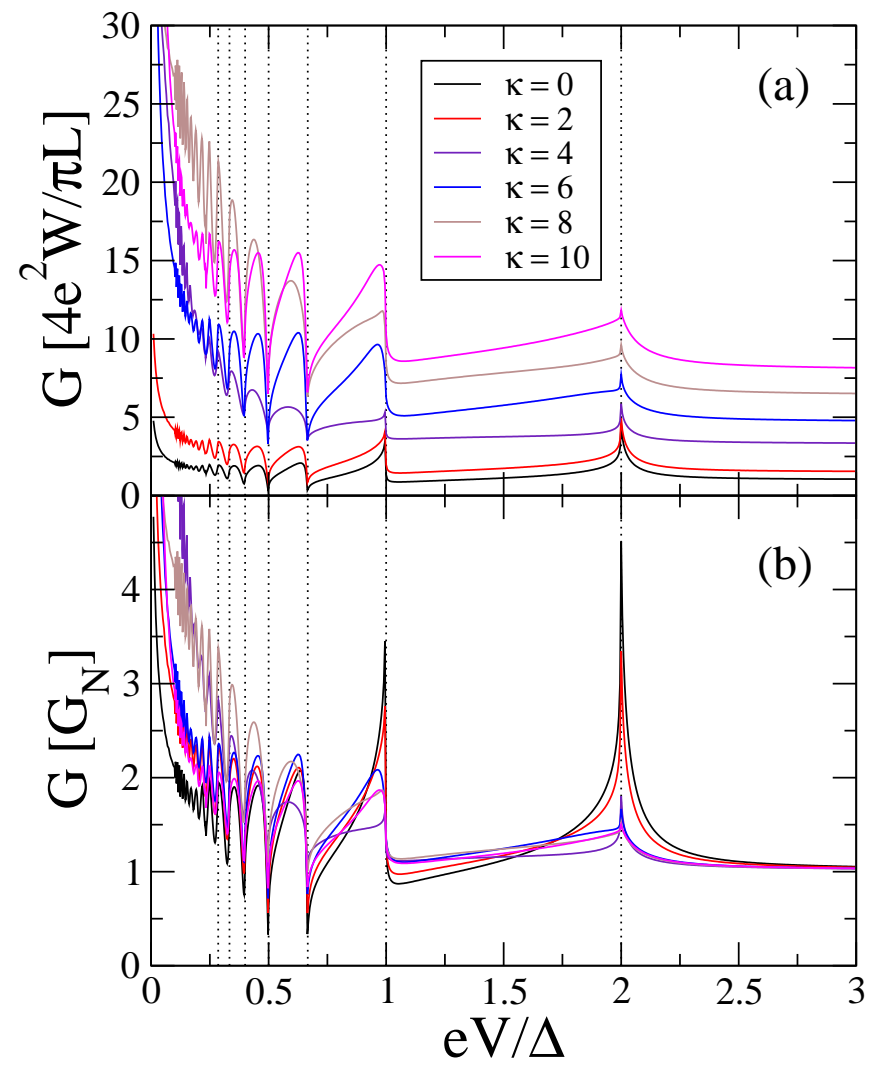

FIG. 3: (Color online) Differential conductance corresponding to the I-V curves of Fig. 2 In panel (b) the conductance is normalized by the normal-state conductance $G_{\mathrm{N}}$. As a guide for the eyes, the dotted vertical lines indicate the position of the voltages $2 \Delta / n e(n=1,2, \ldots, 7)$.

to the Dirac point (see Fig. П1). At very low bias, the conductance exhibits a square-root singularity $(1 / \sqrt{V})$, which originates from the contribution of the highlytransmitting channels ${ }^{12}$. In an actual experiment, this singularity would be masked by the transition to the supercurrent branch at low bias.

Another interesting quantity for a possible comparison with experiments is the so-called excess current, $I_{\text {exc }}$. At voltages $\mathrm{eV} \gg 2 \Delta$ the current can be expressed as $I(V)=I_{\mathrm{N}}(V)+I_{e x c}$, where $I_{\mathrm{N}}((V)$ is the current in the normal state and $I_{e x c}$ is the excess current, which is independent of the voltage. In the case of a single-channel point-contact, the zero-temperature excess current as a function transmission coefficient $\tau$ can be written as ${ }^{10}$

$$
I_{e x c}=\frac{2 e \Delta}{h} \frac{\tau^{2}}{1-\tau}\left[1-\frac{\tau^{2}}{2(2-\tau) \sqrt{1-\tau}} \ln \left(\frac{1+\sqrt{1-\tau}}{1-\sqrt{1-\tau}}\right)\right] .
$$

Averaging this expression with the transmission function for the graphene junction, as indicated in Eq. 目 one obtains the excess current shown in Fig. [4 The gate modulation of $I_{e x c}$ is very similar to the modulation of the critical current found in Ref. 6 . Again, at the Dirac point one recovers the result of a short diffusive SNS junction ${ }^{12.15}$, 


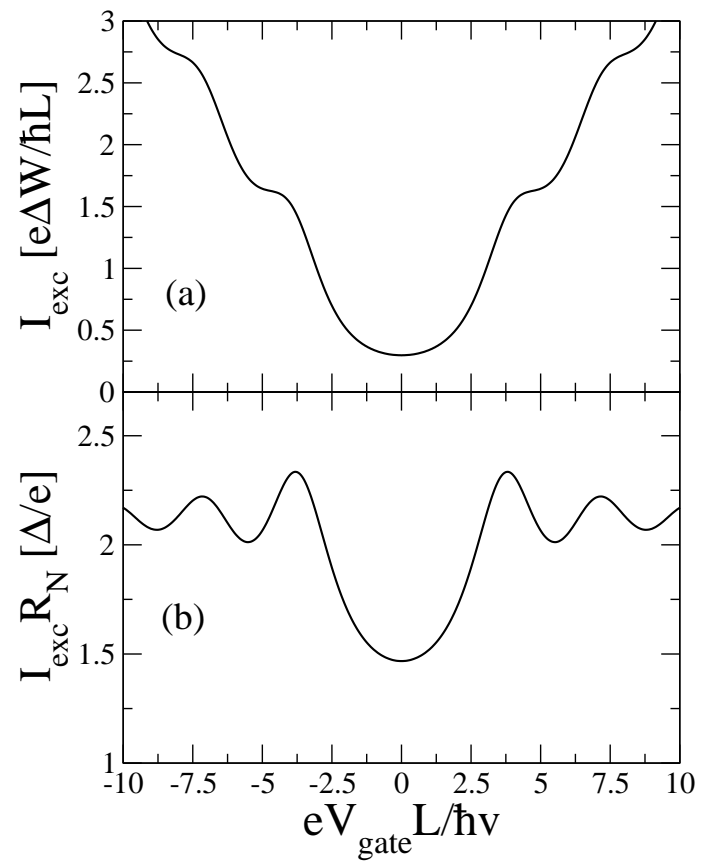

FIG. 4: (a) Zero-temperature excess current as function of the gate voltage for a short ballistic graphene SNS junction. (b) The same as in panel (a), but normalized by the resistance of the junction in the normal state $R_{\mathrm{N}}$.

i.e. $e I_{e x c} R_{\mathrm{N}} / \Delta=\left(\pi^{2} / 4\right)-1$.
Discussion and conclusions.- In this work we have focused our attention in the analysis of the I-V curves of short SNS graphene junctions, but the idea of combining the transmission function of Eq. 2 with the singlechannel point-contact results can be also used to study other transport properties of these junctions such as shot noise ${ }^{16,17}$, Shapiro steps ${ }^{18}$, photon-assisted tunneling ${ }^{18}$ or even full counting statistics 19.20 . For the special case of undoped graphene $\left(V_{\text {gate }}=0\right)$ the transport properties are identical to those of short diffusive SNS systems, which have been already reported in the literature $17,20,21$. Moreover, following the recent work of Katsnelson ${ }^{22}$, a similar idea could be used to study superconducting contacts involving bilayer graphene.

In summary, we have shown that the I-V characteristics of a short ballistic graphene SNS junction exhibits a very rich and gatable subharmonic gap structure. This very pronounced and sensitive structure predicted here can be straightforwardly compared with experimental results in submicron scale junctions ${ }^{4}$ and it might constitute an ideal test quantity to confirm some of the peculiar consequences of the interplay of superconductivity and relativistic quantum dynamics.

Acknowledgments.- We acknowledge discussions with Hubert B. Heersche, Pablo Jarillo-Herrero, Sebastián Bergeret and Elsa Prada. This work has been financed by the Spanish CYCIT (contract FIS2005-06255) and by the Helmholtz Gemeinschaft (contract VH-NG-029).
1 K. S. Novoselov, A. K. Geim, S. V. Morozov, D. Jiang, Y. Zhang, S. V. Dubonos, I. V. Grigorieva, and A. A. Firsov, Science 306, 666 (2004).

${ }^{2}$ K. S. Novoselov, A. K. Geim, S. V. Morozov, D. Jiang, M. I. Katsnelson, I. V. Grigorieva, S. V. Dubonos, and A. A. Firsov, Nature 438, 197 (2005).

3 Y. Zhang, Y.-W. Tan, H. L. Stormer, and P. Kim, Nature 438, 201 (2005).

${ }^{4}$ H.B. Heersche and P. Jarillo-Herrero, private communication.

${ }^{5}$ C. W. J. Beenakker, Phys. Rev. Lett. 97, 067007 (2006).

${ }^{6}$ M. Titov and C. W. J. Beenakker, Phys. Rev. B 74, 041401 (2006).

7 P. Joyez, D. Vion, M. Götz, M. H. Devoret, and D. Esteve, J. Supercond. 12, 757 (1999).

8 T.M. Klapwijk, G.E. Blonder, and M. Tinkham, Physica B 109\&110, 1657 (1982).

9 E.N. Bratus, V.S. Shumeiko, and G. Wendin, Phys. Rev. Lett. 74, 2110 (1995). D. Averin and A. Bardas, Phys. Rev. Lett. 75, 1831 (1995).

10 J.C. Cuevas, A. Martín-Rodero, and A. Levy Yeyati, Phys. Rev. B 54, 7366 (1996).

11 E. Scheer, P. Joyez, D. Esteve, C. Urbina, and M.H. Devoret, Phys. Rev. Lett. 78, 3535 (1997). E. Scheer, N. Agraït, J.C. Cuevas, A. Levy Yeyati, B. Ludoph, A.
Martín-Rodero, G. Rubio, J.M. van Ruitenbeek, and C. Urbina, Nature 394, 154 (1998).

12 A. Bardas and D.V. Averin, Phys. Rev. B 56, R8518 (1997).

13 J. Tworzydło, B. Trauzettel, M. Titov, A. Rycerz, and C. W. J. Beenakker, Phys. Rev. Lett. 96, 246802 (2006).

14 Yu. V. Nazarov, Phys. Rev. Lett. 73, 134 (1994).

15 S.N. Artemenko, A.F. Volkov, and A.V. Zaitsev, Zh. Eksp. Teor. Fiz. 76, 1816 (1979) [Sov. Phys. JETP bf 49, 924 (1979)].

16 J.C. Cuevas, A. Martín-Rodero, and A. Levy Yeyati, Phys. Rev. Lett. 82, 4086 (1999).

17 Y. Naveh and D.V. Averin, Phys. Rev. Lett. 82, 4090 (1999).

18 J.C. Cuevas, J. Heurich, A. Martín-Rodero, A. Levy Yeyati, and G. Schön, Phys. Rev. Lett. 88, 157001 (2002).

19 J.C. Cuevas and W. Belzig, Phys. Rev. Lett. 91, 187001 (2003).

20 G. Johansson, P. Samuelsson, and A. Ingerman, Phys. Rev. Lett. 91, 187002 (2003).

21 J.C. Cuevas and W. Belzig, Phys. Rev. B 70, 214512 (2004).

22 M.I. Katsnelson, cond-mat/0606611 